\title{
Open carpal tunnel release: Comparison of a long versus short incision
}

\author{
Vicki L Kruger MS OT(C), Morris TM Rebot MD FRCSC \\ Queensway General Hospital, Etobicoke, Ontario
}

\section{VL Kruger, MTM Rebot. Open carpal tunnel release: Comparison of a long versus short incision. Can J Plast Surg 1998;6(2):89-92.}

\begin{abstract}
A total of 225 adults with carpal tunnel release of 313 wrists were studied to compare the outcome of a long (at least $3.5 \mathrm{~cm}$ ) with that of a short ( $2.0 \mathrm{~cm}$ or less) incision technique. The two groups were compared for postoperative complaints, length of time until full function was regained, rate of referral to rehabilitation and the effect of Workers' Compensation status. The surgical technique and rehabilitation protocol are described. The number and severity of postoperative complaints were significantly reduced in the short incision group. In the non-Workers' Compensation group, 96\% of short incision patients resumed full function within 28 days This finding compares favourably with published results for endoscopic release, and is superior to results obtained with the long incision. Workers' Compensation patients required longer to recuperate, with $78 \%$ regaining full function within 28 days. Of those with Workers' Compensation, $47 \%$ of the long incision group and $27 \%$ of the short incision group required out-patient therapy. For the non-Workers' Compensation group, this percentage decreased to $21 \%$ of those with the long incision and $3 \%$ with the short incision. A history of vocational or avocational repetitive motion patterns was the most common indicator for therapy and accounted for $67 \%$ of rehabilitation referrals.
\end{abstract}

Key Words: Adult, Carpal tunnel release, Median nerve, Occupational therapy, Physical therapy, Treatment outcome

\section{Dégagement ouvert du canal carpien : comparaison d'une incision longue versus courte}

RÉSUMÉ : En tout, 225 adultes ayant subi un dégagement du canal carpien, totalisant 313 poignets, ont été étudiés afin de comparer les résultats d'une technique d'incision longue (au moins $3,5 \mathrm{~cm}$ ) versus courte $(2,0 \mathrm{~cm}$ ou moins). Les deux groupes ont été comparés au chapitre des plaintes postopératoires, du temps de retour à un fonctionnement normal, du taux de demandes auprès des programmes de réadaptation et de l'effet sur la compensation par la CSST. La technique chirurgicale et le protocole de réadaptation sont décrits. Le nombre et la gravité des problèmes postopératoires ont été significativement moindres dans le groupe ayant subi l'incision courte. Pour le groupe non inscrit à la CSST, $96 \%$ des patients ayant subi une incision courte ont retrouvé un fonctionnement normal en 28 jours. Ces résultats se comparent favorablement aux résultats publiés au sujet du dégagement endoscopique et sont supérieurs aux résultats obtenus avec l'incision longue. Les patients inscrits à la CSST ont pris plus de temps à récupérer, $78 \%$ ayant retrouvé une fonction normale en 28 jours. Parmi les malades assujettis à la CSST, $47 \%$ de ceux qui avaient subi l'incision longue et $27 \%$ de ceux qui avaient subi l'incision courte ont dû être suivis en externe. Pour le groupe non inscrit à la CSST, ce pourcentage a diminué à $21 \%$ de ceux ayant subi une incision longue et à $3 \%$ de ceux ayant subi l'incision courte. Des antécédents de mouvements répétitifs liés ou non au travail ont été l'indicateur le plus fréquent du recours au traitement et ont représenté $67 \%$ des demandes de programme de réadaptation.

A review of our patients who had been treated with open carpal tunnel release (OCTR) undertaken in 1991 revealed that $78 \%$ had an uneventful course of recovery, resuming full function within two to four weeks, and that $25 \%$

Correspondence: Ms V Kruger, 24 Dunboyne Court, Willowdale, Ontario M2R 2B7. Telephone 416-259-6671 ext 5077, e-mail vkruger@compuserve.com required out-patient rehabilitation due to the persistence and magnitude of their symptoms three to four weeks postoperatively. Resumption of activities of daily living (ADL) was delayed by weakness, peri-incisional tenderness, restricted range of movement and swelling.

In an effort to decrease postoperative morbidity and shorten recovery time, our surgical protocol was re-examined. Decreased postoperative discomfort and shorter recovery 


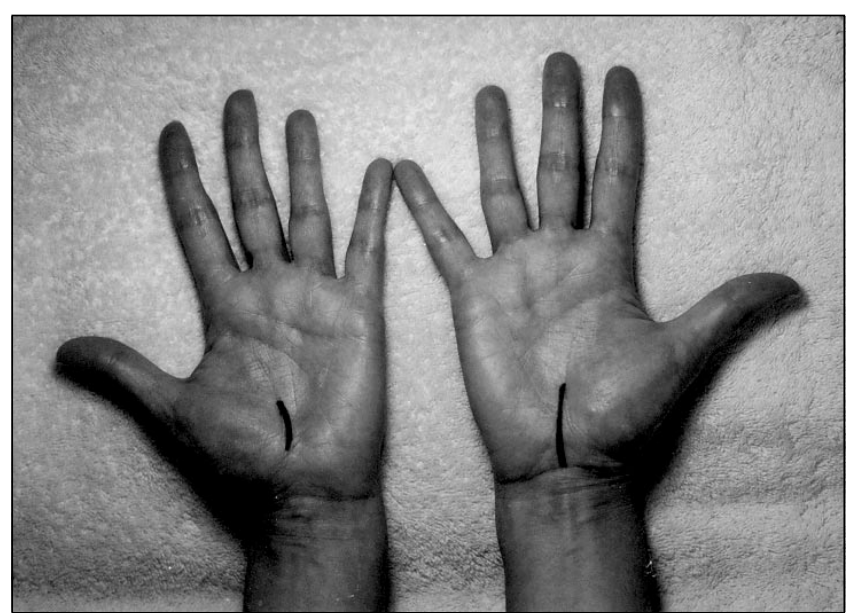

Figure 1) Representation of the short and long incisions made

time are benefits ascribed to endoscopic carpal tunnel release (ECTR) (1-3). Unfortunately, the cost of the instruments proved prohibitive. To determine whether we could obtain the benefits of endoscopy but with instruments on hand, we commenced using a shorter incision with the open technique in 1992. The long (4) and short incisions used are illustrated in Figure 1.

A six-year retrospective study was subsequently conducted to compare the outcome of the long (approximately $3.5 \mathrm{~cm}$ ) versus short (approximately $2.0 \mathrm{~cm}$ ) incisions, and to profile those patients likely to require rehabilitation.

\section{Patients}

\section{PATIENTS AND METHODS}

Consecutive medical records of 237 patients who had undergone carpal tunnel release (CTR) by the same plastic surgeon between April 1989 and July 1995 were reviewed. The 225 patients eligible for inclusion in the study had carpal tunnel syndrome, which had been electrodiagnostically confirmed in 377 wrists. Twelve patients were excluded because their etiology included direct trauma to the median nerve or carpus. Sixty-nine patients underwent CTR in 95 hands using the long incision; 156 underwent CTR in 218 hands using the short incision, for a total of 313 procedures. If both carpal tunnels required release, the second was not done until functional independence had been regained.

Data collected included age, sex, length of time that symptoms were present before CTR, type of insurance coverage and concomitant conditions. Concomitant conditions were classified as none; inflammatory and degenerative joint disorders; indirect trauma (ie, upper limb fracture, crush, laceration or inflammation not in proximity to the carpus); endocrine abnormalities (ie, diabetes mellitus or hypothyroidism); known vocational or avocational stress (ie, rapid repetitive movements or use of vibratory tools); or other. The category 'other' included any disorder that may affect body fluid volume or tone (ie, cardiac, respiratory, renal or neurological).

The rate of referral for rehabilitation was analyzed. Groups were compared by presenting complaints and the number of weeks of follow-up required before full function was regained.

\section{Surgical technique (short incision)}

To facilitate accurate closure at the end of the procedure, multiple transverse lines are placed across the distal portion of the thenar crease line (ulnar-most thenar crease line if more than one). A $2.0 \mathrm{~cm}$ incision through skin only, exposing the underlying fat, is carried out distal to the confluence of the thenar and hypothenar pads. The underlying fat is then swept aside (without incising) to the level of the palmar fascia to avoid injury to branches of the palmar cutaneous nerve. Senn retractors (V Mueller, Illinois) are employed to expose the ulnar portion of the palmar fascia in line with the axis of the ring finger. A longitudinal incision through the palmar fascia exposes the underlying distal portion of the transverse carpal ligament (TCL). In this position, any variation of the anatomy at the distal portion of the TCL can be examined. Sharp scalpel incision into the mid to distal ulnar portion of the TCL a few millimetres radial to the hook of the hamate allows the exposure of the underlying contents, usually exposing the flexor tendons. The distal portion of the remaining TCL is released with blunt dissection using sharp point scissors. The remaining proximal portion of the TCL is approached by first passing blunt tip instrumentation superficial to the contents of the carpal tunnel and on the deep surface of the TCL to free any possible adhesions to the TCL. Blunt narrow tipped scissors are then slightly opened and placed at a right angle to include the TCL within the opening. The scissors are then gently pushed in the proximal direction to release the remaining intact proximal TCL and the distal $2 \mathrm{~cm}$ portion of the antebrachial fascia in the wrist region.

Further exploration of the contents of the carpal tunnel is next done to rule out any anatomical variations that may be creating physical blocking (ie, muscle, lipoma or ganglions). If anatomical variation occurs, the incision may be extended in the usual fashion to complete the necessary dissection. Skin closure only, using the previous multiple transverse lines as an accurate guide for reapposition, is then done. A bulky hand dressing leaving all digits exposed and freely mobile is applied and left in place for 10 to 14 days. Gentle activity of the hand is encouraged while the hand is in dressing.

\section{Rehabilitation protocol}

Two to four weeks: All patients were seen in the Ambulatory Care Clinic (ACC) 10 to 14 days postoperatively for removal of the surgical dressing and sutures. The occupational therapist provided education, reassurance and a home program of range of motion exercises, edema management techniques and recommendations for functional hand use. If the patient expressed anxiety or did not feel he or she could resume ADL, one to three additional visits were scheduled to monitor progress and answer specific questions. Rarely, a patient expressed such anxiety or magnified symptoms that they were referred directly to therapy.

Five to seven weeks: If full functional independence had not been regained within four weeks, patients were referred for 


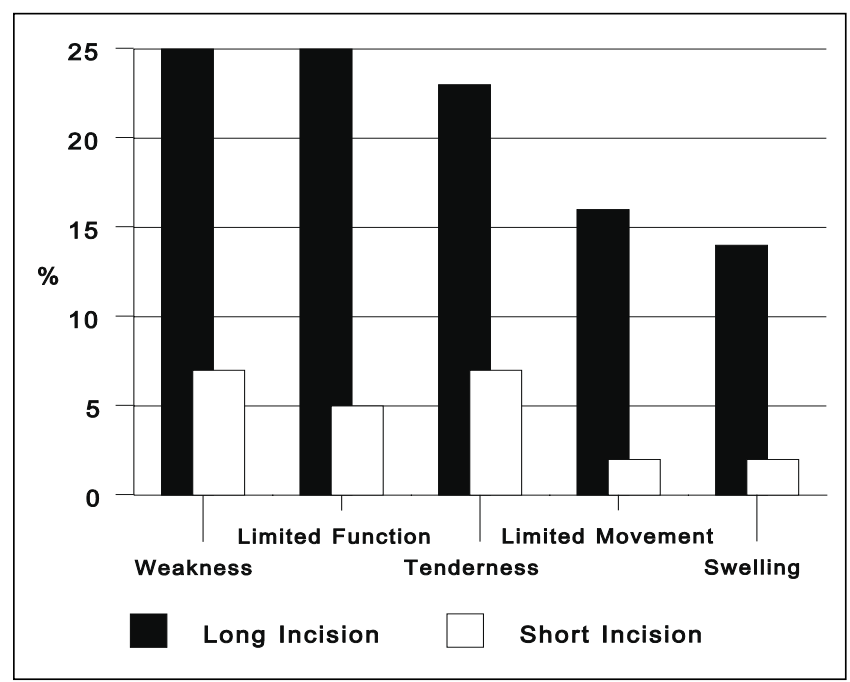

Figure 2) Postoperative complaints in the two groups

out-patient physiotherapy and occupational therapy, which consisted of attendance at a $1 \mathrm{~h}$ hand class three times per week for a course of ultrasound and/or laser, contrast baths, progressive resistive exercises and, when needed, recommendations for ergonomic modifications to the home or job site. The sole criterion for class membership was a diagnosis of injury or disease of the upper limb, distal to the elbow; type of insurance reimbursement was not a factor. Sessions averaged 10 to 15 patients each, with a spectrum of stages of recovery represented. A class format was selected for the benefits of the group dynamics; group members help, encourage and motivate each other, and problems and coping mechanisms are shared with others who have undergone the same procedure but are at a different stage in the recovery process $(5,6)$. The group serves as a gauge of patient's behaviour, which, when combined with a spirit of friendly competition, has a favourable impact on recovery time. In addition, use of a group format virtually eliminated a waiting list for therapy.

Eight to 10 weeks: For those with persistent scar tenderness (usually accompanied by weakness), a palmar splint (Smith \& Nephew Rolyan Inc, Wisconsin) with a shock-absorbing gel insert was fitted to protect the scar line during hand use. This splint did not restrict wrist or finger movement and allowed more aggressive hand use. When necessary, return to a modified work program was organized with the employer.

After 10 weeks: Those who had not regained full function after 10 weeks were referred to their physician for further investigation. They were invariably diagnosed with proximal or systemic concomitant conditions.

\section{RESULTS}

Patients ranged from 18 to 89 years old; median age was 58 years. Fifty-seven per cent were female. Sixty-two per cent had been symptomatic for more than one year, $30 \%$ for four to 12 months and $8 \%$ for less than three months. Eleven per cent of the patient population had no known concomitant conditions, $26 \%$ had some form of arthritis, $23 \%$ had other

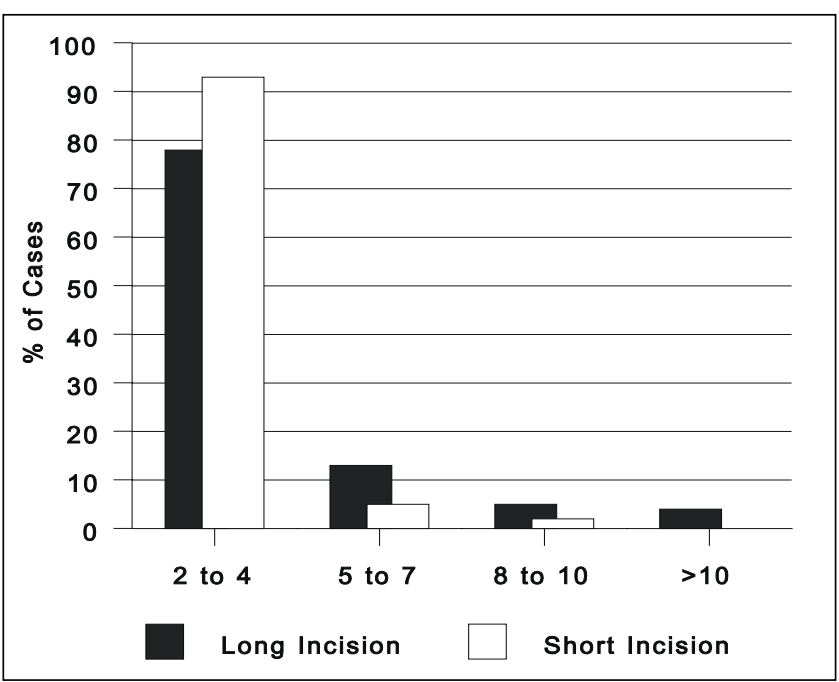

Figure 3) Number of weeks to regain full function in the two groups

medical conditions, $20 \%$ had vocational or avocational stress, $13 \%$ had endocrine abnormalities and $7 \%$ had indirect trauma. Eighty-three per cent (187 of 225) of the patients were covered by the Ontario Health Insurance Plan (OHIP) and $17 \%$ (38 of 225) were receiving Workers' Compensation (WC). There were no statistically significant differences between the long and short incision groups for age, sex, length of time that symptoms were present before CTR, concomitant conditions or insurance coverage. Two patients in the long incision group (both with insulin-dependent diabetes mellitus) experienced delayed wound closure due to the development of infection. No surgical complications were noted in the short incision group.

Postoperative complaints of the two groups are compared in Figure 2. Improvement was noted in all areas in the short incision group and was statistically significant at $\mathrm{P} \leq 0.05\left(\chi^{2}\right)$.

The rate of referral for out-patient therapy was determined using $\chi^{2}$ analysis. Of those who underwent CTR using the long incision, $25 \%$ were referred ( 24 of 95 hands in 22 patients). This referral rate decreased to $8 \%$ for those who received the short incision (17 of 218 hands in 14 patients). This was significant at $\mathrm{P} \leq 0.05$. Of those with OHIP coverage, $21 \%$ (17 of 80 hands) of the long incision group and 3\% (six of 177 hands) of the short required therapy. Of those with WC coverage, this increased to $47 \%$ (seven of 15 hands) of the long group and 27\% (11 of 41 hands) of the short.

As outlined in Figure 3, the two groups were compared for length of follow-up required before full function was regained. The short incision group regained function in significantly less time $\left(\mathrm{P} \leq 0.05, \chi^{2}\right)$.

\section{DISCUSSION}

Ninety-three per cent of our patients who underwent OCTR with the short incision resumed regular ADL within 28 days. This finding compares favourably with ECTR results for the resumption of ADL, for which studies report a range of $60.4 \%$ to $85.9 \%$ within 28 days $(3,7,8)$, a mean of 20.7 to 39.8 days $(7,9,10)$ or a median of 14 to 25 days $(11,12)$. 
Two studies reported on a similar short OCTR technique. Hallock and Lutz (9) obtained a mean return to function time of 46.3 days for the total group - 22.6 days for non-WC cases and 59.5 days for WC cases. Nathan et al (13) reported better results using a program of early aggressive physical therapy. They described a median of 12 days to return to function for the total group - 10 for non-WC cases and 18 for WC cases.

When a comparison was made by type of insurance reimbursement, patients who received WC required longer to recuperate and were more likely to require therapy than those who did not $(7,9,10,12-17)$. Ninety-seven per cent of our short incision OHIP patients (171 of 177 hands) resumed ADL within 28 days. Reported ECTR results range from $78.3 \%$ to $100 \%$ within 28 days $(7,8,14)$, a mean of 10.8 to 22.3 days $(7,9,10,14,15)$ or a median of 13 to 16.5 days $(12,14)$. Seventy-eight per cent of our short incision WC population ( 32 of 41 hands) resumed ADL within the 28-day period. Other studies reported a range of $33 \%$ to $63 \%$ within 28 days $(7,8,14)$, a mean of 29.2 to 57.6 days $(7,9,10,14,15)$ or a median of 57 to 71 days $(12,14)$. The above studies were conducted in the United States, where many authors suspect that secondary gain is a factor in the recovery of WC patients $(12,13,16,17)$. In Ontario, the Workers' Compensation Act (18) obligates employers to reinstate injured workers in their previous job or a suitable alternative, whenever possible. Early return to work is encouraged and the New Experimental Experience Rating program (19) provides financial incentives to employers to accommodate this.

\section{REFERENCES}

1. Okutsu I, Ninomiya S, Takatori Y, Ugawa Y. Endoscopic management of carpal tunnel syndrome. Arthroscopy 1989;5:11-8.

2. Chow JCY. Endoscopic release of the carpal ligament: A new technique for carpal tunnel syndrome. Arthroscopy 1989;5:19-24.

3. Chow JCY. Endoscopic release of the carpal ligament for carpal tunnel syndrome: 22-month clinical result. Arthroscopy 1990;6:288-96.

4. Heckler FR, Jabaley ME. Evolving concepts of median nerve decompression in the carpal tunnel. Hand Clin 1987;2:723-36.

5. Versluys H. Psychological adjustment to physical disability. In: Trombly CA, Scott AD, eds. Occupational Therapy for Physical Dysfunction. Baltimore: Waverly Press, 1977:24-6.

6. Posthuma BW. The small group in counseling and therapy. In: Small Groups in Counseling and Therapy: Process and Leadership, 2nd edn. Needham Heights: Allyn \& Bacon, 1996:5-7.

7. Palmer DH, Paulson JC, Lane-Larsen CL, Peulen VK, Olson JD. Endoscopic carpal tunnel release: A comparison of two techniques with open release. Arthroscopy 1993;9:498-508.

8. Chow JCY. The Chow technique of endoscopic release of the carpal ligament for carpal tunnel syndrome: Four years of clinical results. Arthroscopy 1993;9:301-14.

9. Hallock GG, Lutz DA. Prospective comparison of minimal incision open and two-portal endoscopic carpal tunnel release. Plastic Reconstr Surg 1995;96:941-7.

10. Roth JH, Richards RS, MacLeod MD. Endoscopic carpal tunnel release. Can J Surg 1994;37:189-93.
The patient most likely to require rehabilitation presented with a need for repetitive motion patterns, regardless of WC status. We found that $67 \%$ of these out-patients were employed in jobs requiring the use of power tools, machines or cleaning implements. An additional 17\% comprised those with indirect trauma. Arthritis and endocrine abnormalities accounted for the remaining $16 \%$ of referrals. Out-patient follow-up was not required for those with no concomitant conditions or those in the 'other' category.

The typical patient who required more than eight weeks of follow-up (for one hand) reported bilateral symptoms, whether or not both carpal tunnels had been released. They were also employed in a job where no light duties were available to them.

\section{CONCLUSIONS}

The efficacy of a short incision OCTR is comparable with that reported for endoscopic CTR and is accomplished with standard instrumentation. Outcomes for the short incision OCTR are superior to those reported for the long incision OCTR. Selective referral to hand therapy seems to shorten the recovery period for patients at risk.

ACKNOWLEDGEMENTS: The authors express appreciation to Liina Veer OT(C) whose support and assistance made this study possible. Thanks also to Lawrence Milne MD for invaluable editorial advice, Barbara Wilkins RN for her assistance in the retrieval of data and Kai-Lii Veer for the photograph.

11. Brown RA, Gelberman RH, Seiler JG, et al. Carpal tunnel release: A prospective, randomized assessment of open and endoscopic methods. J Bone Joint Surg 1993;75A:1265-75.

12. Agee JM, McCarroll HR Jr, Tortosa RD, Berry DA, Szabo RM, Peimer CA. Endoscopic release of the carpal tunnel: A randomized prospective multicenter study. J Hand Surg 1992;17A:987-95.

13. Nathan PA, Meadows KD, Keniston RC. Rehabilitation of carpal tunnel surgery patients using a short surgical incision and an early program of physical therapy. J Hand Surg 1993;18A:1044-50.

14. Nagle D, Harris G, Foley M. Prospective review of 278 endoscopic carpal tunnel releases using the modified Chow technique. Arthroscopy 1994;10:259-65.

15. Kerr CD, Gittins ME, Sybert DR. Endoscopic versus open carpal tunnel release: Clinical results. Arthroscopy 1994;10:266-9.

16. Higgs PE, Edwards D, Martin DS, Weeks PM. Carpal tunnel surgery outcomes in workers: Effect of workers' compensation status. J Hand Surg 1995;20A:354-60.

17. Nagle D. Endoscopic carpal tunnel release: In favor. Clin Plast Surg 1996;23:477-86.

18. Ontario Government. Revised Statutes of Ontario 1990. Obligation To Re-Employ. In: Workers' Compensation Act, Chapter W.11, Section 54. Toronto: Queen's Printer for Ontario.

19. The New Experimental Experience Rating. NEER User Guide. Toronto: Workplace Safety \& Insurance Board, 1996:2-8. 